\title{
Über eine neue Klasse von Elektrolyten in verflüssigtem Schwefeldioxyd
}

\author{
Von Fritz Seel und Hans Bauer
}

Aus dem Anorganisch-chemischen Institut der Technischen Hochschule München

(Z. Naturforschg. 2 b, 397-400 [1947]; eingegangen am 8. August 1947)

\begin{abstract}
Die Säureradikale der Carbonsäuren - RCO - und salpetrigen Säure - NO - können in verflüssigtem Schwefeldioxyd als selbständige (solvatisierte) Kationen - $\mathrm{RCO}^{+}$, NO+ - auftreten, wie dies von Metall-, Hydronium- und Ammoniumionen in wäßrigen Systemen bekannt ist. Dies ist dadurch möglich, daß man den entsprechenden Säurehalogeniden - RCOCl, $\mathrm{NOCl}$ - durch Zusatz elektrophiler Komplexbildner, wie $\mathrm{SbCl}_{5}$, $\mathrm{AlCl}_{3}$, das Halogen als Anion entziehen kann.

Es werden die Ergebnisse neuer Leitfähigkeitsuntersuchungen an solchen Systemen mitgeteilt sowie charakteristische „Ionenreaktionen“ dieser neuartigen Elektrolyte beschrieben.
\end{abstract}

$\mathrm{I}^{\mathrm{n}}$ m Rahmen seiner Untersuchungen in nichtwäßrigen Lösungsmitteln hat bereits P. Walde ${ }^{1}$ gefunden, daß verflüssigtes Schwefeldioxyd als Lösungsmittel ein ausgesprochen gutes Ionisierungsmittel ist, und neuerdings konnte dies insbesondere durch die systematischen Arbeiten von G. J a nder ${ }^{2}$ über die „Chemie in flüssigem Schwefeldioxyd" bestätigt werden. Viele Salze gehen in ihm unter Dissoziation in solvatisierte Ionen (bzw. Ionenkomplexe) in Lösung, ja selbst in ungelöstem Zustande als Nichtelektrolyte bekannte Stoffe können in verflüssigtem Schwefeldioxyd in Ionen gespalten werden. Wenn auch die für wäßrige Systeme charakteristische Säuredissoziation infolge der mangelnden Ausbildungsmöglichkeit von Lyoniumionen ${ }^{3}$ fehlt, so besitzt andererseits das flüssige Schwefeldioxyd bei gewissen konstitutiven Voraussetzungen der entsprechenden gelösten Verbindungen die Fähigkeit zur Ionentrennung der Kohlenstoff-Halogen-Bindung, welche in Wasser - ohne gleichzeitig stattfindende solvolytische Prozesse - durchaus nicht

1 Vergl. z. B. P. Wa l d e n, Ber. dtsch. chem. Ges. 32. 2862 [1899]; Z. anorg. allg. Chem. 30, 145 [1902]; Z. physik. Chem. 42, 432 [1905].

${ }_{2}$ Vergl. z. B. G. J a n d e r, Naturwiss. 26, 779, 793 [1938]; neueste Arbeit: Z. anorg. allg. Chem. 250, 304 [1943].

3 Unter „Lyonium“-Ionen werden solche Kationen verstanden, welche sich durch Anlagerung eines Protons an das Lösungsmittel-Molekül, bilden, z. B.

$$
\mathrm{OH}_{2}+\mathrm{H}^{+} \rightarrow \mathrm{OH}_{3}^{+}, \mathrm{NH}_{3}+\mathrm{H}^{+} \rightarrow \mathrm{NH}_{4}^{+} \text {. }
$$

möglich ist. So ist bereits früher bekannt geworden, daß Triarylmethylchloride ${ }^{4}$ sowie gewisse ungesättigte Ketochloride ${ }^{5}$ in flüssigem Schwefeldioxyd den elektrischen Strom leiten; man hat Grund, als Ursache hierfür die Abdissoziation von Chlorionen anzunehmen:

$$
\begin{aligned}
\mathrm{RCl} \rightarrow \mathrm{R}^{+}+\mathrm{Cl}^{-} ; \quad \mathrm{R}^{+} \text {z. B. }=\left(\mathrm{C}_{6} \mathrm{H}_{5}\right)_{3} \mathrm{C}^{+} \\
\quad \text { oder } \quad \mathrm{CH}_{3} \mathrm{O} \cdot \mathrm{C}_{6} \mathrm{H}_{4} \cdot \mathrm{CH}: \mathrm{CH} \cdot \stackrel{+}{\mathrm{C}} \mathrm{Cl} \cdot \mathrm{C}_{6} \mathrm{H}_{4} \cdot \mathrm{OCH}_{3} .
\end{aligned}
$$

Vor einigen Jahren ${ }^{6}$ konnte gezeigt werden, daß in dem genannten Lösungsmittel eine weitere Klasse organischer Halogenverbindungen, nämlich die der Carbonsäurehalogenide - ebenso wie auch Nitrosylchlorid - , Halogenionen abspalten kann, wenn diese Ionentrennung durch Zusatz elektrophiler Komplexbildner - insbesondere z. B. Antimon(V)-chlorid - gefördert wird. Es schließt sich nämlich hierbei das abgetrennte Halogenion an dessen Atomverband unter Bildung des Anions der entsprechenden Halogenosäure an $\left(\mathrm{Cl}^{-}+\mathrm{SbCl}_{5} \rightarrow\left[\mathrm{SbCl}_{6}\right]\right)$, so daß Lösungen der Acylderivate - besser Acyl-Salze der Halogenosäuren entstehen, welche sich von dem zugesetzten komplexbildenden Halogenid ableiten, z. B.

${ }^{4} \mathrm{~K}$. Z i e gle r u. H. Wohlschütt, Liebigs Ann. Chem. 479, 90 [1930].

${ }^{5}$ F. Str a u \& u. A. D ü t z m an n, J. prakt. Chem. 103, 1 [1921].

6 F. S e e l, Z. anorg. allg. Chem. 250, 331 [1943]; 252, 24 [1943]. 


$$
\begin{aligned}
\mathrm{CH}_{3} \mathrm{COCl}+\mathrm{SbCl}_{5} & \rightarrow \mathrm{CH}_{3} \mathrm{CO}^{+}+\left[\mathrm{SbCl}_{6}\right]^{-} ; \\
\mathrm{C}_{6} \mathrm{H}_{5} \mathrm{COCl}+\mathrm{SbCl}_{5} & \rightarrow \mathrm{C}_{6} \mathrm{H}_{5} \mathrm{CO}^{+}+\left[\mathrm{SbCl}_{6}\right]^{-} ; \\
\mathrm{NOCl}+\mathrm{SbCl}_{5} & \rightarrow \mathrm{NO}^{+}+\left[\mathrm{SbCl}_{6}\right]^{-} .
\end{aligned}
$$

Es ist bemerkenswert, daß sämtliche der betrachteten Kationen mesomeriefähige Systeme enthalten:

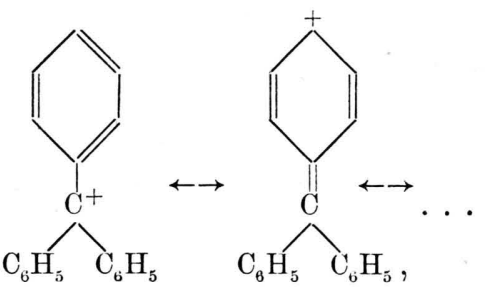

$$
\begin{aligned}
& \mathrm{CH}_{3} \mathrm{O} \cdot \mathrm{C}_{6} \mathrm{H}_{4} \cdot \mathrm{CH}: \mathrm{CH} \cdot \stackrel{+}{\mathrm{C} C l} \cdot \mathrm{C}_{6} \mathrm{H}_{4} \cdot \mathrm{OCH}_{3} \\
& \leftrightarrow \mathrm{CH}_{3} \mathrm{O} \cdot \mathrm{C}_{6} \mathrm{H}_{4} \cdot \stackrel{+}{\mathrm{C}} \mathrm{H} \cdot \mathrm{CH}: \mathrm{CCl} \cdot \mathrm{C}_{8} \mathrm{H}_{4} \cdot \mathrm{OCH}_{3} \\
& \mathrm{R}-\stackrel{+}{\mathrm{C}}=\underline{\mathrm{O}}|\leftrightarrow \mathrm{R}-\mathrm{C} \equiv \stackrel{+}{\mathrm{O}}|, \quad|\stackrel{+}{\mathrm{N}}=\underline{\mathrm{O}}| \leftrightarrow|\mathrm{N} \equiv \stackrel{+}{\mathrm{O}}| .
\end{aligned}
$$

Bei den Carbonsäureradikal- und Nitrosyl-Ionen ist eine Mesomerie zwischen Carbenium- bzw. Azeniumund Oxonium-Valenzzustand ${ }^{7}$ möglich. Offenbar deckt die „Stabilisierungsenergie“ des Mesomerieeffektes einen Teil der Ionentrennungsarbèit der ElementHalogen-Atombindung.

Die begonnenen orientierenden Untersuchungen am "Acetylfluoborat", $\left(\mathrm{CH}_{3} \mathrm{CO}\right)\left[\mathrm{BF}_{4}\right]$, und den in den Gln. (1) bis (3) angeführten Acyl-chloroantimonaten(V) sind inzwischen fortgesetzt worden.

Besonders eindrucksvoll läßt sich der in (1) bis (3) gegebene Vorgang durch eine Titration vorgelegter Mengen Säurechlorid in flüssigem Schwefeldioxyd mit Antimon(V)-chlorid bei gleichzeitigem Verfolgen des Leitfähigkeitswertes der Lösung reproduzieren. Die aus den Leitfähigkeitswerten erhaltenen Kurven (vgl. Abb.1) zeigen ausgesprochen scharfe Knickpunkte beim Molverhältnis 1:1 und jenseits desselben praktisch keine Veränderung der Leitfähigkeit. Dies bedeutet, daß die in Frage kommenden Gleichgewichte von dem Überschuß der einen Komponente - im Bereich der gewählten experimentell noch bedeutsamen Konzentration - unabhängig sind, also unter diesen Umständen ganz auf der Seite der Acylsalzbildung liegen.

7 ,Valenzzustände“ sind jene (nicht stationären) Zustände eines Moleküls, welche durch die einzelnen ,Valenzformeln“ mit lokalisierten Bindungen angedeutet werden können und durch deren Überlagerung (Mesomerie) erst die eigentlichen stationären $\mathrm{Zu}$ stände entstehen.
$\mathrm{Zu}$ demselben Ergebnis führen auch genauere Leitfähigkeitsmessungen von Lösungen verschiedener Konzentrationen, welche aus äquivalenten Mengen Acetyl-, Benzoyl- oder Nitrosylchlorid und Antimon(V)-chlorid bzw. direkt aus den bestehenden festen Verbindungen $\mathrm{CH}_{3} \mathrm{COCl}+\mathrm{SbCl}_{5}$, $\mathrm{C}_{6} \mathrm{H}_{5} \mathrm{COCl}+\mathrm{SbCl}_{5}, \quad \mathrm{NOCl}+\mathrm{SbCl}_{5}$ hergestellt sind $^{8}$. Im Gebiet stärkerer Konzentrationen besitzt der Wert der molaren Leitfähigkeit dieser Verbindungen die Größenordnung starker Elektrolyte, wie von Tetramethyl-ammoniumperchlorat $\left(\left[\mathrm{N}\left(\mathrm{CH}_{3}\right)_{4}\right] \mathrm{ClO}_{4}\right)$ oder des in diesem Zusammen-

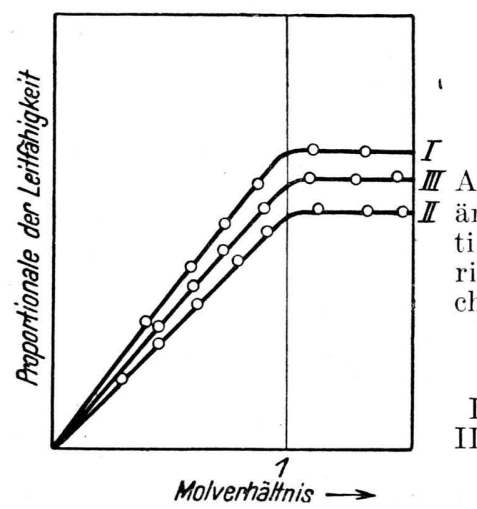

Abb. 1. Leitfähigkeitsänderungen bei Titrationen von Säurechloriden mit Antimon(V) chlorid in etwa $0,02-m$. Lösung.

I $\mathrm{CH}_{3} \mathrm{COCl}+\mathrm{SbCl}_{5}$ II $\mathrm{NOCl}+\mathrm{SbCl}_{5}$ III $\mathrm{C}_{6} \mathrm{H}_{5} \mathrm{COCl}+\mathrm{SbCl}_{5}$

hang besonders interessierenden Kaliumsalzes der Antimon(V)-chlorwasserstoffsäure, $\mathrm{KSbCl}_{6}$ (vgl. Abb. 2).

Dadurch, daß die Verbindungen $\mathrm{NOCl}, \mathrm{CH}_{3} \mathrm{COCl}$ $+\mathrm{SbCl}_{5}$ in konzentrierteren Lösungen eine molare Leitfähigkeit besitzen, welche größer ist als der halbe Betrag der Leitfähigkeit eines schwach assoziierten starken Elektrolyten, wird die Annahme von Vorgängen der Art $2 \mathrm{SbCl}_{5}+2 \mathrm{CH}_{3} \mathrm{COCl} \rightarrow\left[\mathrm{Sb}_{\left(\mathrm{ClCOCH}_{3}\right)_{2}}^{\mathrm{Cl}_{4}}\right]^{+}$ $\left[\mathrm{SbCl}_{6}\right]^{-}$, also die Bildung von ,Einlagerungsverbindungen" (welche mit dem Ergebnis der Titration vereinbar wären) ausgeschlossen.

Während die molare Leitfähigkeit bei normalen Elektrolyten (z. B. $\left.\left[\mathrm{N}\left(\mathrm{CH}_{3}\right)_{4}\right] \mathrm{ClO}_{4}\right)$ infolge des geringer werdenden Assoziationsgrades und der größeren Beweglichkeit der Ionen mit abnehmender Konzentration größer wird, tritt bei den untersuchten Elektrolyten nach Erreichung eines Maximums der Leitfähigkeit wieder eine Verringerung derselben ein bzw. erfolgt deren Anstieg bereits wesentlich langsamer als bei den normalen

8 Diese Verbindungen sind bereits früher isoliert worden. Vgl. H. M e e r w e in u. H. M a i e r-H ü s e r, J. prakt. Chem. 134, 68, 69 [1922]; H. R h e i n b o ld u. R. Was s e r u hr, Ber. dtsch. chem. Ges. 60, $732[1927]$. 
Elektrolyten. Es ist hiernach anzunehmen, daß mit steigender Verdünnung entweder die Zahl der vorhandenen Ionen geringer wird, oder daß Elektrolyte mit kleinerer Äquivalentleitfähigkeit gebildet werden. Letzteres ist beim Alkalisalz der Antimonchlorwasserstoffsäure der Fall, so daß der Charakter seiner Leitfähigkeitskurve durch das Gleichgewicht $\mathrm{K}\left[\mathrm{SbCl}_{6}\right] \rightleftarrows \mathrm{KCl}+\mathrm{SbCl}_{5}$ bestimmt wird ${ }^{9}$. Da die Ausgangskomponenten der untersuchten Acylsalze, Säurechloride und Antimon (V)-chlorid, in flüssigem Schwefeldioxyd innerhalb unseres Konzentrationsbereiches nur

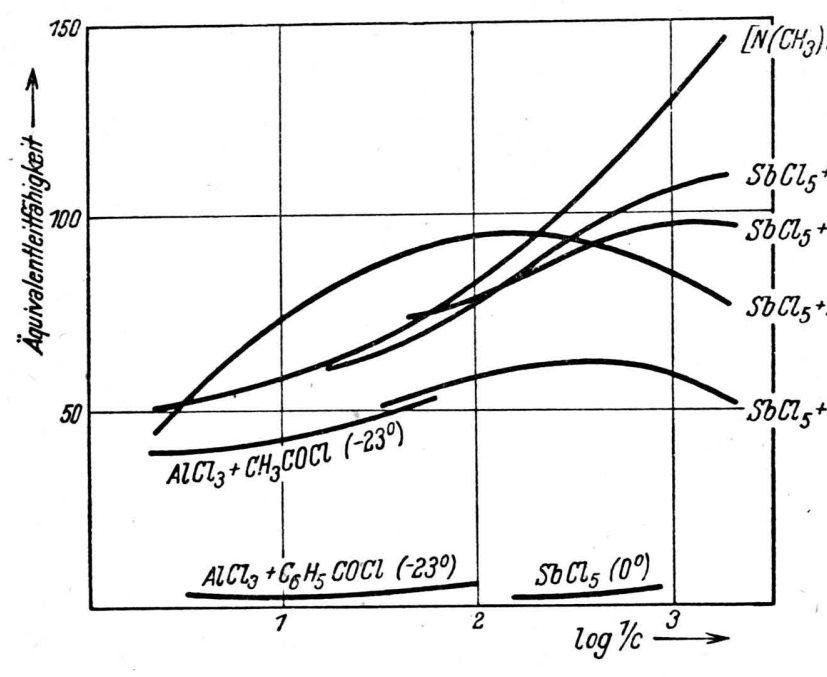

Abb. 2. Äquivalentleitfähigkeitswerte in $\mathrm{cm}^{2} \Omega^{-1}$ in flüssigem Schwefeldioxyd.

sehr schwache Elektrolyte sind (weil sie nur in geringem Maße dissoziieren), ist wohl anzunehmen, daß das Abfallen der Äquivalentleitfähigkeit der Komplexe mit abnehmender Konzentration ebenfalls auf der Rückbildung der Komponenten beruht:

$$
\begin{gathered}
\mathrm{AcSbCl}_{6} \rightarrow \mathrm{AcCl}+\mathrm{SbCl}_{5} ; \\
\mathrm{Ac}=\mathrm{CH}_{3} \mathrm{CO}, \mathrm{C}_{6} \mathrm{H}_{5} \mathrm{CO}, \mathrm{NO},
\end{gathered}
$$

d.h. daß die in konzentrierteren Lösungen vorhandenen Ionenassoziate ${ }^{10}\left[(\mathrm{RCO})\left(\mathrm{SbCl}_{6}\right)\right]^{x-y}$ in die wenig dissoziierten Komponenten RCOCl und $\mathrm{SbCl}_{5}$ und nicht in Einzelionen $\mathrm{RCO}^{+}$und $\mathrm{SbCl}_{6}^{-}$aufgelöst werden. Es läßt sich aus diesen

${ }^{9}$ Vergl. G. J a nder u. H. M esech, Z. physik. Chem., Abt. A, 183, 255 [1939].

10 Die stark ausgeprägte Ionenassoziation ist ein Charakteristikum des Lösungsmittels $\mathrm{SO}_{2}$, welches bei weitem nicht die depolymerisierende Kraft des Wassers besitzt.

${ }_{11}$ H. Ulich u. Nespital, Z. angew. Chem. 44, 750 [1921].
Verhältnissen auch schließen, daß der extrem assoziierte feste Zustand der Verbindungen $\mathrm{RCOCl}+\mathrm{SbCl}_{5}$ dem von Salzen $\mathrm{RCO}\left[\mathrm{SbCl}_{6}\right]$ entspricht und nicht etwa Koordinationsverbindungen wie $\mathrm{Cl}_{5} \mathrm{Sb} \ldots \mathrm{ClCOR}$ oder $\mathrm{Cl}_{5} \mathrm{Sb} \ldots$ OCClR .

Da Aluminiumchlorid in verflüssigtem Schwefeldioxyd sehr stabile Solvate (z. B. $\mathrm{AlCl}_{3} \cdot 2 \mathrm{SO}_{2}$ ) bildet, ist es weniger als Acylsalzbildner $\left(\mathrm{AcCl}+\mathrm{AlCl}_{3} \rightarrow \mathrm{Ac}\left[\mathrm{AlCl}_{4}\right]\right)$ geeignet wie Antimon(V)-chlorid. So zeigt schon die Lösung $\mathrm{AlCl}_{3}+\mathrm{C}_{6} \mathrm{H}_{5} \mathrm{COCl}$ kaum ein stärkeres Leitvermögen als die Einzelkomponenten. Im ungelösten Zustande bestehen diese Unterschiede zwischen $\mathrm{AlCl}_{3}$ und $\mathrm{SbCl}_{5}$ jedoch nicht, so daß auch die kristalline Verbindung $\mathrm{C}_{6} \mathrm{H}_{5} \mathrm{COCl}+\mathrm{AlCl}_{3}$ die Struktur eines Salzes $\left[\mathrm{C}_{6} \mathrm{H}_{5} \mathrm{CO}\right]^{+}\left[\mathrm{AlCl}_{4}\right]^{-}$ haben dürfte. Hierfür spricht auch deren hohe Dielektrizitätskonstante ${ }^{11}$.

Die, wenn auch geringe, so doch immerhin endliche spezif. Leitfähigkeit der Säurechloride $\mathrm{CH}_{3} \mathrm{COCl}, \mathrm{C}_{6} \mathrm{H}_{5} \mathrm{COCl}, \mathrm{NOCl}^{12}$ in flüssigem Schwefeldioxyd, welche die eines Nichtelektrolyten erheblich überschreitet und bei dem Verdünnungsgrad $50(0,02 \mathrm{Mol} / l)$ etwa eine Zehnerpotenz größer ist als die des Lösungsmittels, weist auf ein Dissoziationsgleichgewicht $\mathrm{AcCl} \rightleftarrows \mathrm{Ac}^{+}$ $+\mathrm{Cl}^{-}$hin, das praktisch allerdings ganz auf der linken Seite liegt. Ebenso wird die schwache Leitfähigkeit des Antimon(V)-chlorids durch ein Gleichgewicht etwa der $\mathrm{Art} \mathrm{SbCl}_{5} \rightleftarrows \mathrm{SbCl}_{4}{ }^{+}+\mathrm{Cl}^{-} \mathrm{zu}$ erklären sein. (Für eine Ionisierung desselben spricht auch seine Fällbarkeit durch Sulfit ${ }^{13}$.)

Die elektrolytische Dissoziation der Acylchlorantimonate wie $\mathrm{CH}_{3} \mathrm{CO}\left[\mathrm{SbCl}_{6}\right]$ usw. in verflüssigtem Schwefeldioxyd ermöglicht durch Zusatz von Fremdelektrolyten die Durchführung von Ionenreaktionen, wie sie aus der Chemie in wäßrigen Lösungen bekannt sind. Es sind solche sowohl im präparativen Maßstab durchgeführt wie auch konduktometrisch verfolgt worden. Durch Messung der Leitfähigkeitsänderungen während der Reaktionen kann in den Fällen, wo das neu entstehende Acylderivat in flüssigem Schwefeldioxyd löslich ist, auf dessen Konstitution, d. h. seine Elektrolytbzw. Nichtelektrolytnatur, geschlossen werden. So ergeben die Umsetzungen

$$
\begin{gathered}
\mathrm{CH}_{3} \mathrm{CO}\left[\mathrm{SbCl}_{6}\right]+\left[\mathrm{N}\left(\mathrm{CH}_{3}\right)_{4}\right] \mathrm{ClO}_{4} \\
2 \mathrm{CH}_{3} \mathrm{CO}\left[\mathrm{SbCl}_{6}\right]+\left[\mathrm{N}\left(\mathrm{CH}_{3}\right)_{4}\right]_{2} \mathrm{SO}_{4},
\end{gathered}
$$

und

12 Vergl. F. S e e l, Anm. 6.

13 G. J a n d e r, Z. anorg. allg. Chem. 250, 296 [1943]. 
daß die Carbonsäureradikalderivate der Überchlor- und Schwefelsäure keine Acylsalze bzw. in flüssigem Schwefeldioxyd nur sehr schwache Elektrolyte sind (vergl. Abb. 3):

$$
\begin{gathered}
\mathrm{CH}_{3} \mathrm{CO}^{+}+\left[\mathrm{ClO}_{4}\right]^{-} \rightarrow \mathrm{CH}_{3} \mathrm{CO} \cdot \mathrm{OClO}_{3}, \\
2 \mathrm{CH}_{3} \mathrm{CO}^{+}+\left[\mathrm{SO}_{4}\right]^{-} \rightarrow\left(\mathrm{CH}_{3} \mathrm{CO} \cdot \mathrm{O}\right)_{2} \mathrm{SO}_{2} .
\end{gathered}
$$

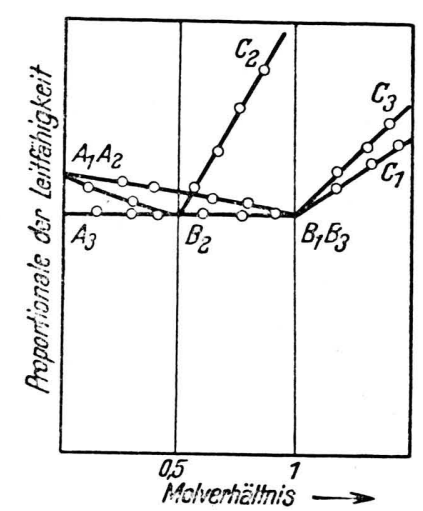

Abb. 3. Leitfähigkeitsänderungen bei Titrationen von Acyl-chlorantimonaten mit Tetramethylammoniumperchlorat und -sulfat in etwa $0,02-m$. Lösung.

$\mathrm{A}_{1} \mathrm{~B}_{1} \mathrm{C}_{1} \quad \mathrm{CH}_{3} \mathrm{CO}\left[\mathrm{SbCl}_{6}\right]+\left[\mathrm{N}\left(\mathrm{CH}_{3}\right)_{4}\right] \mathrm{ClO}_{4}$, $\mathrm{A}_{2} \mathrm{~B}_{2} \mathrm{C}_{2} \quad \mathrm{CH}_{3} \mathrm{CO}\left[\mathrm{SbCl}_{6}\right]+\left[\mathrm{N}\left(\mathrm{OH}_{3}\right)_{4}\right]_{2} \mathrm{SO}_{4}$, $\mathrm{A}_{3} \mathrm{~B}_{3} \mathrm{C}_{3} \mathrm{NO}\left[\mathrm{SbCl}_{6}\right]+\left[\mathrm{N}\left(\mathrm{CH}_{3}\right)_{4}\right] \mathrm{ClO}_{4}$.

Deutung des Kurvenverlaufs:

$\mathrm{A}_{1} \mathrm{~B}_{1}$ Bildung von nicht dissoziiertem $\mathrm{CH}_{3} \mathrm{CO} \cdot \mathrm{OClO}_{3}$ - Umwandlung von $\mathrm{CH}_{3} \mathrm{CO}\left[\mathrm{SbCl}_{6}\right]$ in $\mathrm{NR}_{4}\left[\mathrm{SbCl}_{6}\right]$

$\mathrm{A}_{2} \mathrm{~B}_{2}$ Bildung von nichtdissoziiertem $\left(\mathrm{CH}_{3} \mathrm{CO} \cdot \mathrm{O}\right)_{2} \mathrm{SO}_{2}$ - Umwandlung von $\mathrm{CH}_{3} \mathrm{CO}\left[\mathrm{SbCl}_{6}\right]$ in $\mathrm{NR}_{4}\left[\mathrm{SbCl}_{6}\right]$,

$\mathrm{A}_{3} \mathrm{~B}_{3}$ Bildung von nicht löslichem $\mathrm{NO}\left[\mathrm{ClO}_{4}\right]-\mathrm{Um}$ wandlung von $\mathrm{NO}\left[\mathrm{SbCl}_{6}\right]$ in $\mathrm{NR}_{4}\left[\mathrm{SbCl}_{6}\right]$,

$\mathrm{B}_{1} \mathrm{C}_{1}, \mathrm{~B}_{3} \mathrm{C}_{3}$ Überschuß von $\mathrm{NR}_{4} \mathrm{ClO}_{4}, \mathrm{~B}_{2} \mathrm{C}_{2}$ Überschuß von $\left(\mathrm{NR}_{4}\right)_{2} \mathrm{SO}_{4}$.
Bei der Einwirkung von $\left[\mathrm{N}\left(\mathrm{CH}_{3}\right)_{4}\right] \mathrm{ClO}_{4}$ auf $\mathrm{NO}\left[\mathrm{SbCl}_{6}\right]$ liegt der Fall vor, wo ein in flüssigem Schwefeldioxyd unlösliches Salz - Nitrosylperchlorat $\mathrm{NO}\left[\mathrm{ClO}_{4}\right]-$ gebildet wird ${ }^{14}$ :

$$
\mathrm{NO}^{+}+\left[\mathrm{ClO}_{4}\right]^{-} \rightarrow\left[(\mathrm{NO})^{+}\left(\mathrm{ClO}_{4}\right)^{-}\right]_{\text {fest }} .
$$

Auch Acetylperchlorat ist ebenso wie Acetylchlorid in flüssigem Schwefeldioxyd in sehr geringem Maße, und zwar in dem sinne $\mathrm{CH}_{3} \mathrm{CO} \cdot \mathrm{OClO}_{3} \rightarrow \mathrm{CH}_{3} \mathrm{CO}^{+}$ $+\mathrm{ClO}_{4}^{-}$dissoziiert, wodurch es ebenfalls Ionenreaktionen eingehen kann: so fällt KJ $\mathrm{KClO}_{4}$, NOCl $\mathrm{NOClO}_{4}$. Interessanterweise kann dagegen aus einer Lösung des Nitrosylderivates der Reinecke-Säure mittels $\mathrm{N}\left(\mathrm{CH}_{3}\right)_{4} \mathrm{ClO}_{4}$ kein $\mathrm{NOClO}_{4}$ gefällt werden. Es ist demnach nicht deren Nitrosylsalz - wie bisher vermutet worden ist -, sondern ein Derivat des Nitrosylrhodanids: $\left[\mathrm{Cr}\left(\mathrm{NH}_{3}\right)_{2}(\mathrm{NCS})_{3} \mathrm{NCS} \cdot \mathrm{NO}\right]$. Hiermit ist auch dessen hohe Hydrolysenbeständigkeit verständlich.

Die Durchführung der Untersuchungen an den extrem feuchtigkeitsempfindlichen Stoffen stellte große Anforderungen an die apparative Gestaltung, besonders der Gefäße zur Messung der Leitfähigkeiten. Es wurde hierzu eine besondere Hochvakuumtechnik für das Arbeiten mit tiefsiedenden Lösungsmitteln unter völligem Ausschluß von Luft und Feuchtigkeit entwickelt, über welche gelegentlich einer ausführlichen Besprechung dieser und weiterer Versuche berichtet werden wird.

Hrn. Prof. Dr. W. H i e b e r danken wir bestens für die Förderung dieser Arbeit durch Überlassung von Institutsmitteln.

14 Ebenso läßt sich mittels $\mathrm{N}\left(\mathrm{CH}_{3}\right)_{4} \mathrm{SO}_{4} \mathrm{H}$ Nitrosylschwefelsäure, $\mathrm{NOSO}_{4} \mathrm{H}$, gewinnen. Durch konsequente Weiterentwicklung solcher Umsetzungen konnten neuerdings eine Reihe bisher unbekannter interessanter Nitrosylderivate dargestellt werden.

\title{
Über ungewöhnliche Lichtabsorption einiger einfacher Indophenolfarbstoffe
}

\section{Von Heinz Schulze*}

\author{
(Z. Naturforschg. 2 b, 400-404 [1947]; eingegangen am 23. August 1947)
}

\begin{abstract}
In der Gruppe der in der Regel blauen bis grünen Indophenole aus 1.2-Phenolcarbonsäureamiden und Phenylendiaminen wurden neue, sämtlich im Amidrest mit stark polaren Gruppen substituierte Farbstoffe gefunden, deren Absorptionsbanden in den Bereich kürzerer. Wellenlängen verschoben sind. Als Ursache für die Verschiebung werden Nebenvalenzkräfte angenommen.
\end{abstract}

$\mathrm{B}$ ei mehreren modernen Farbenfilmverfahren ${ }^{1}$, bei denen das Bild aus den drei subtraktiven Grundfarben Gelb, Purpur und Blaugrün aufge-

* Z. Zt. Kaiser-Wilhelm-Institut für Biochemie, Tübingen, Gmelinstr. 8. baut wird, besteht der blaugrüne Bildfarbstoff aus einem Indophenol. Er wird bei der Entwicklung des belichteten Films nach $\mathrm{F}$ is che $\mathrm{r}^{2} \mathrm{z}$. B. nach

\footnotetext{
1 E. St en g e r u. H. St a u d e, Fortschr. d. Photographie, Band II u. III, Leipzig 1942 u. 1943.
}

2 DRP. 253335. 